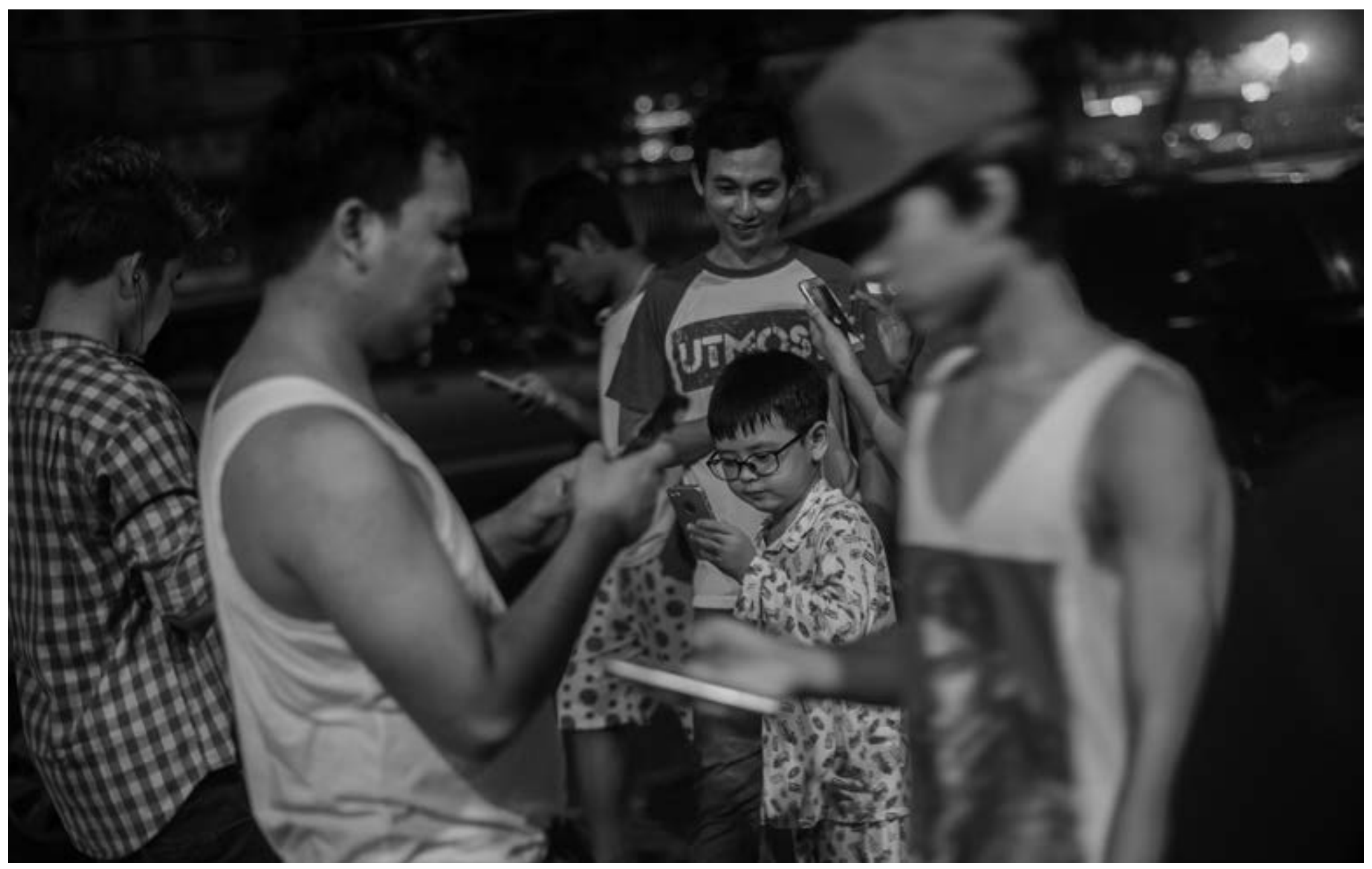

Foto: ${ }^{\oplus A F P / Y e ~ A u n g ~ T h u . ~ Y a n g o n, ~ a g o s t o ~ d e ~} 2016$

\title{
El sujeto en el centro de la socialización mediática: entre la recepción y la participación virtual
}

The place of the subject in media socialization: between reception and virtual participation

https://doi.org/10.22235/d.v0i25.1273

Salomé Sola-Morales 
Salomé Sola-Morales Universidad de Santiago de Chile Santiago de Chile, Chile salome.sola@usach.cl

Recepción: octubre 2016 Aceptación: noviembre 2016

\section{RESUMEN}

El objetivo de este artículo es reflexionar sobre la condición socializadora de los medios de comunicación en un entorno donde conviven la tradicional recepción mediática y las nuevas formas de participación virtual. Tomando como punto de partida las aportaciones de Alfred Schütz, Albert Bandura Claudio Esteva Fabregat y Lluís Duch, se pone de manifiesto la necesidad de profundizar en el concepto de socialización mediática y en el rol clave de los sujetos. Para ahondar en este proceso se debe adoptar una perspectiva dialéctica que integre el rol de los seres humanos como entes mediadores y la capacidad socializadora de los propios discursos mediáticos, dejando a un lado cualquier visión pasiva de los sujetos.

Palabras clave: socialización, medios de comunicación, interacción social, mediación, antropología cultural

\section{Introducción}

Este artículo reflexiona acerca de la condición socializadora de los medios de comunicación y coloca en el centro del análisis a los sujetos y a los grupos como entes mediadores. La socialización mediática ha sido estudiada en extenso desde los estudios de comunicación (Boulier, 1991; Gerbner, Gross, Morgan y Signorelli, 1986; Potter, 1996; Signorelli, 1990; 1993; Silverstone, 2004; Wolf, 1992). Muchos investigadores se han centrado en el impacto de los medios sobre los adolescentes y niños (Durham, 1999; McDevitt y Chaffee, 2000; Chapin, 2000; Fisherkeller, 2002; 0'Keefe y Reid-Nash, 1987; Pasquier, 1996; Pindado, 2003, 2005; Suess, Suoninen, Garitaonandia, Juaristi, Koikkalainen y Oleaga, 1998; Walkerdine, 1993), los efectos sobre la vida familiar (Chaffee, McLeod y Walkman, 1973; Fujioka y Austin, 2002; Lull, 1980; Silverstone, Hirsh y Morley, 1996), sobre los roles de género (Collins, 2011; Dill y Till, 2007; Dietz, 1998; Hardin y Greer, 2009;

\section{ABSTRACT}

The aim of this article is to reflect on the socializing condition that media have in a context in which coexist the traditional reception and new forms of virtual participation. Taking as starting point the contributions of Alfred Schütz, Albert Bandura, Claudio Esteva Fabregat and Lluís Duch it is evident the need to deepen the concept of media socialization and the key role of subjects. Therefore, it is necessary to adopt a dialectical perspective that integrates the role of humans as mediators and the socializing capacity of media discourses, leaving behind all passive vision of subjects.

Keywords: socialization, media, social interaction, mediation, cultural anthropology

Gross, 1991; Hartmann y Klimmt, 2006) o el agente y consumidor (Barber, 2014; Bush, Smith y Martin, 2013; Wang, Yu y Wei, 2012).

El alcance de los medios de comunicación en la diseminación de valores y conocimientos se ha trabajado mucho desde una perspectiva comunicativa. No obstante, se ha profundizado poco en el fenómeno de la socialización mediática desde un enfoque holístico, que integre los aportes de diferentes tradiciones teóricas. En este artículo se reflexiona entonces sobre la necesidad de entender la socialización mediática desde la perspectiva de los sujetos como entes mediadores (Martín Serrano, 1987; Sola-Morales, 2013b). No se trata ya de un efecto de los contenidos, los relatos o los fenómenos mediáticos sobre los individuos o los grupos, sino de una interacción- socialización que transita en un doble sentido o doble movimiento: desde los medios hacia a los sujetos y desde los sujetos hacia los medios. 
Esta perspectiva dialéctica resulta esclarecedora en la era de Internet, donde los sujetos dejaron de ser entes pasivos que reciben el impacto de los medios. Por el contrario, son sujetos activos que utilizan los medios como plataformas de socialización, comunicación y debate para transformar la realidad misma. Esto se evidencia en la creación de redes sociales en torno a la sexualidad o el embarazo, por ejemplo, o en el desarrollo de movimientos sociales o procesos políticos a través de la red, tales como el 15M en España o la primavera árabe, por citar dos casos paradigmáticos. Esta propuesta toma en cuenta el rol de los medios, pero, sobre todo, focaliza en la condición mediadora de los sujetos y pretende devolver el rol protagónico al ser humano, relegado en parte por el mensaje o el canal.

Aunque la estructura socializadora de los medios de comunicación pueda considerarse una premisa aceptada e incluso obvia, es necesario profundizar en este concepto para dotarlo de mayor precisión. No se puede generalizar y aceptar que las formas de socialización mediática que se dan en los entornos virtuales son las mismas que las que se daban al calor de la radio o la televisión en el entorno familiar cuando solo existían estos medios, dado que no había interactividad posible. No obstante, a un nivel estructural, independiente de los canales y los formatos a través de los cuales se produzca la comunicación, la socialización es una condición existencial del ser humano. La socialización aquí es entendida en un sentido amplio, como un fenómeno natural para todos los individuos, que les facilita convivir en sociedad. La socialización mediática no se trata entonces de un fenómeno exclusivo de los discursos mediáticos, sino una forma natural de mediación que los sujetos y los grupos utilizan para definirse e interactuar con otros.

Es preciso matizar que con el vocablo socialización -distinto de sociabilidad- se designa la aptitud o competencia de los actores para establecer vínculos sociales o interactuar con otros; aptitud que también podría denominarse inteligencia social o aprendizaje social. El antropólogo Miguel Rodríguez afirma que la socialización es:

Un proceso complejo por el cual los individuos aprenden a interiorizar las normas de comportamiento y los valores sociales del grupo o comunidad de pertenencia integrándolos en su personalidad, con el fin de adaptarse a su contexto social (Rodríguez, 1997, p. 87).

Precisamente por ello, los procesos de socialización mediática revisten un interés crucial, pues están en la base de fenómenos como la adquisición de valores, opiniones y creencias, pero también en el desarrollo de relaciones e interacciones como la identificación con los personajes de ficción y no-ficción (Sola-Morales, 2013c) o con personas y grupos en la vida cotidiana. Es más, los medios tienen una función mediadora clave en todas las etapas de la vida (Martín Barbero, 1998; Martín Serrano, 1987).

Para profundizar en la condición mediadora de los sujetos se revisitan cuatro aproximaciones teóricas: el enfoque fenomenológico del sociólogo Alfred Schütz; el abordaje socio-comportamental del psicólogo Albert Bandura; el enfoque culturalista del antropólogo Claudio Esteva Fabregat y el análisis antropológico-filosófico propuesto por el antropólogo Lluís Duch. ¿Por qué esta elección? En primer lugar, porque estos cuatro autores presentan elementos de comprensión claves para el estudio de los medios como instituciones socializadoras que condicionan los procesos de identificación, socialización e interacción de los individuos y los grupos. En segundo lugar, porque cada uno de estos enfoques tienen en común el énfasis que ponen en el vínculo entre la sociedad y el individuo, consideraciones que componen el fundamento general de este estudio. 


\section{El enfoque fenomenológico: Alfred Schütz}

Frente al predominio de las teorías sociológicas estructurales, la propuesta de Alfred Schütz sobre las estructuras del mundo de la vida, los acervos del conocimiento y la socialización es clave para entender el alcance de los medios en la socialización. El autor parte de una sociología interpretativa que trata de comprender la sociedad tanto desde la perspectiva de los sistemas como de los actores (Schütz, 1972b); desde la articulación de lo objetivo hasta lo intersubjetivo (Schütz y Luckmann, 2001). Es decir, desde los sistemas, las estructuras y las instituciones hasta las representaciones que se dan en la vida cotidiana y que son protagonizadas por sujetos y grupos.

El recurso de Schütz al concepto de mundo de la vida es crucial, pues permite entender la co-implicación entre la intersubjetividad y la socialización, dos procesos que son claves para entender la incidencia de los medios en la conducta y en la interacción social entre los individuos. Cabe recordar que para Schütz (1972a) la conducta es como una vivencia de la conciencia, que confiere significado mediante actividad espontánea. Su investigación del mundo de la vida parte de la constatación de que lo social no es algo independiente y externo de sus miembros -individuos o grupos- sino que es el resultado de las prácticas intencionales de estos. En sus palabras:

Una vez supuesta la existencia del tú, ya hemos entrado en el dominio de la intersubjetividad. El individuo vivencia entonces el mundo como algo compartido por sus congéneres, es decir, como un mundo social (Schütz, 1972a, p. 169).

1:: Del griego áutós ('sí mismo') y roínoıc ('creación'), este neologismo es entendido como una condición de existencia de los seres vivos en una continua producción de sí mismos.

Aquí se puede observar la influencia de Weber en el pensamiento de Schütz. Para Weber (1964) no es suficiente que una acción establezca el contacto con otra persona para que se la califique de acción social,
De manera que la acción solo puede comprenderse si se presupone la existencia de ese tú. Cuando dos personas se vuelven recíprocamente orientadas entre sí, se logra lo que Weber llama una relación social. Cabe recordar el carácter intencional e intersubjetivo que el autor da a este concepto:

Por relación social se debe entender un comportamiento de diversos individuos instaurado recíprocamente según su contenido de sentido, y orientado en conformidad. Por tanto, la relación social consiste exclusivamente en la posibilidad de que se obre socialmente de un modo determinado (dotado de sentido), que sea la base en la que descansa tal posibilidad (Weber, 1964, pp. 23-24).

Pero, ¿por qué es relevante la idea del mundo de la vida para comprender mejor la incidencia de los medios en los procesos de socialización y, en concreto, en la convivencia entre formas de recepción y participación virtual? Porque este concepto acentúa la dimensión social en la que se construyen los marcos de sentido que sirven para definir e interpretar la realidad. A saber, remite a procesos y estructuras que posibilitan la comprensión y transforman los estilos de vida y de pensamiento en la vida cotidiana. En este sentido, el mundo creado y representado por los medios de comunicación es un universo de significación, es decir, conforma una urdimbre de sentidos diversos que es preciso interpretar para orientarse y conducirse en él. Pero a un tiempo, y gracias a los nuevos medios sociales, son los sujetos mismos los que crean esos universos de significación, de manera que la interacción social entre sujetos y medios es autopoiética (Maturana y Varela, 1973). estas acciones deben estar enfocadas sobre otro: un tú.
En la participación virtual, los sujetos pueden experimentar, performar o teatralizar nuevas versiones de su yo mediante la creación de avatares o perfiles de usuario en blogs o redes sociales (Sola-Morales, 2013a, p. 93). 


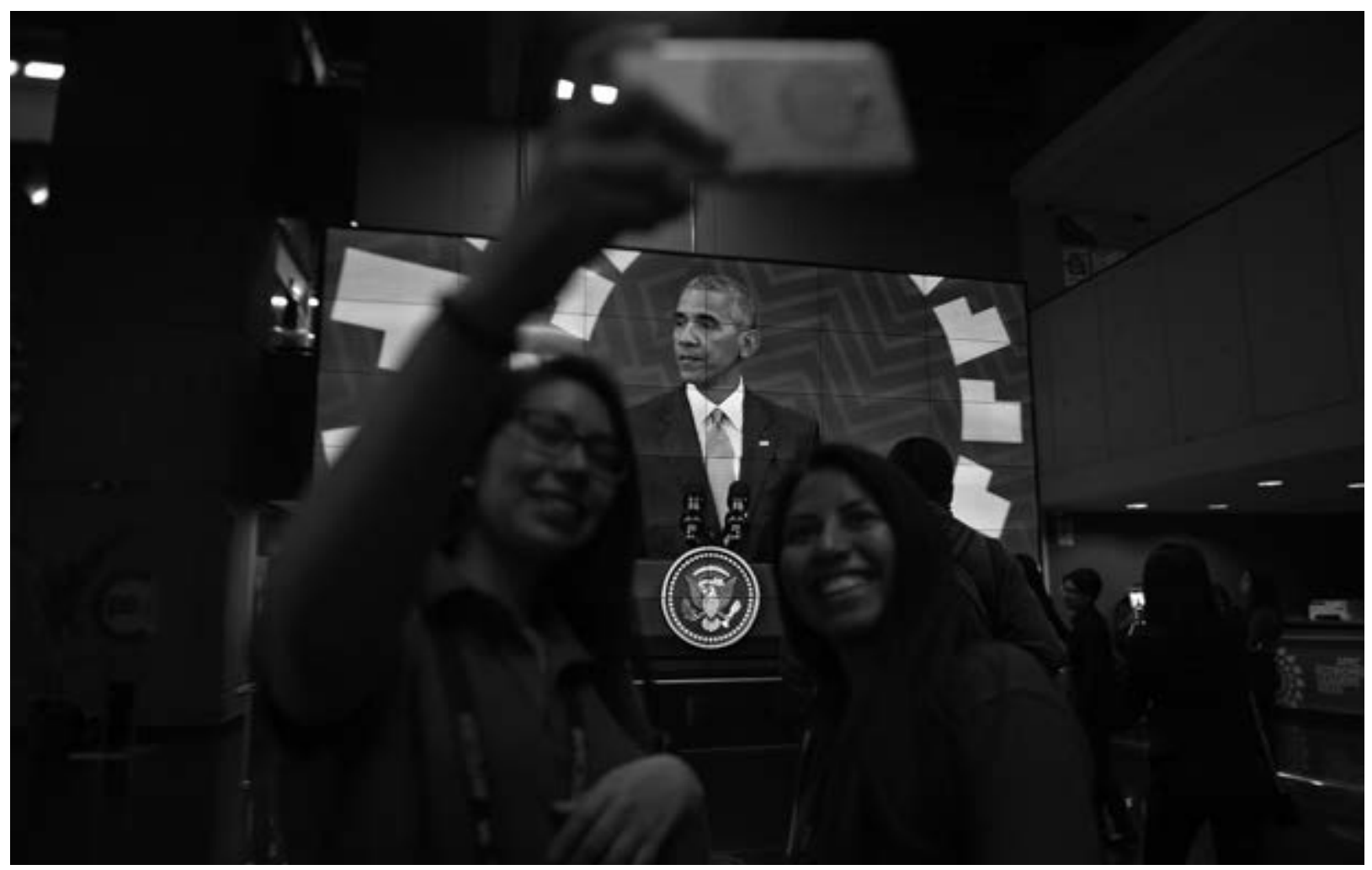

Foto: ๑AFP/Rodrigo Buendía. Lima, noviembre de 2016

Ahora bien, no se puede olvidar que toda reinterpretación del mundo y del yo se basa en un acervo de experiencias, propias o ajenas, que funcionan como esquemas de referencia. Justamente, el concepto de un acervo de conocimiento a mano -también llamado comprensiones del sentido común- es muy útil para explicar este fenómeno. Este concepto alude a una serie de experiencias o percepciones del mundo acumuladas, que permiten tipificar a los otros.

Schütz afirma que, para comprender la conducta de los otros, los sujetos recurren a una suerte de tipificación, por la cual aplican esquemas interpretativos aprendidos, que permiten captar los significados de lo que realizan los otros y ellos mismos. Pero, ¿de dónde provienen estas pautas de aprendizaje social? En gran medida serán instituidas por los relatos que circulan en los medios de comunicación. Vale añadir que en todas las formas de relación social el sí mismo sólo puede ser captado mediante la construcción de una forma típica de conducta, una pauta típica de motivos subyacentes, de actividades típicas, de un tipo de personalidad (Schütz, 2003, pp. 46-47). Tal análisis remite necesariamente al punto de vista subjetivo, es decir, a la interpretación de la acción del otro.

Ahora bien, una dimensión básica del acervo del conocimiento, aparte de su carácter pragmático esencial, es la familiaridad, ya que la mayoría de estos esquemas son compartidos por los miembros de una comunidad. De hecho, gracias a este sustrato significativo común, los individuos acceden al conocimiento del mundo social y pueden construir una realidad social compartida, además de otorgar sentido a su propia biografía. ¿De qué modo adquieren los individuos y los grupos estos esquemas significativos? Como han expresado Peter Berger y Thomas Luckmann, discípulos de Schütz, en su clásica obra La construcción social de la realidad (2008), estos esquemas típicos se conforman y transmiten a través de tres procesos: memoria, imaginarios compartidos y lenguaje. El primero de ellos, la memoria, se constituye gracias a una serie de experiencias estereotipadas en el recuerdo, que permite a los individuos hallar sentido a su biografía. 
El segundo proceso que permite la sedimentación intersubjetiva es la biografía compartida, imaginario o memoria colectiva que es, en gran medida, configurada por los medios de comunicación. El tercer proceso es el lenguaje, generador fundamental de sentidos.

La sedimentación intersubjetiva puede llamarse verdaderamente social solo cuando se ha objetivado en cualquier sistema de signos, o sea, cuando surge la posibilidad de objetivizaciones reiteradas de las experiencias compartidas. Solo entonces hay probabilidad de que esas experiencias se transmitan de una generación a otra, y de una colectividad a otra (Berger y Luckmann, 2008, p. 89).

No obstante, las narraciones propuestas por los medios -0 , si se prefiere, el discurso mediático en toda su amplitud-son en gran parte las responsables de la creación de estas estructuras tipificadoras - de tenor arquetípico, estereotípico y típico- que son adquiridas por los individuos y reutilizadas en sus situaciones de interacción y participación virtual. Así, en cualquier encuentro cara a cara o comunicación mediatizada, el actor utilizará este acervo del conocimiento a mano que le permitirá, como se mencionó, tipificar al otro y socializar con él.

\section{El enfoque socio-comportamental: Albert Bandura}

Este enfoque, que estudia los procesos por los que se adquiere y modifica la conducta social, también es fundamental para comprender la función socializadora de los medios desde una perspectiva psicológica. Albert Bandura, investigador de esta corriente, considera que los medios de comunicación se convierten en un elemento social de gran influencia para aprender y modelar los comportamientos, valores, actitudes y habilidades individuales (Bandura, 2009). Es por ello que los procesos mediáticos de aprendizaje social son una de las fuentes de inspiración más importantes en los procesos de formación del yo.
Desde la perspectiva de la teoría del aprendizaje, gran parte de las experiencias no tienen por qué ser necesariamente experimentadas por el propio individuo, sino que pueden ser observadas en otros de forma directa o indirecta. Tal como explica Bandura (1962, 1977), hay bastantes pruebas de que puede haber aprendizaje por observación, incluso cuando el observador no reproduce las respuestas del modelo durante la adquisición y, por tanto, no recibe refuerzo.

Ahora bien, lo importante en relación al refuerzo vicario - por el cual se modifica la conducta de un observador en virtud del refuerzo administrado al modelo (Bandura y Walters, 1974, p. 18) - es el lugar del modelo en la "jerarquía” (p. 33). Así, estos procedimientos son más eficaces cuando la persona o el agente que los promueve tiene mayor prestigio (en forma de poder, sea ideológico o adquisitivo). Esto explicaría por qué el discurso mediático es tan poderoso y tiene tanta incidencia sobre las personas y los grupos, y sobre la transmisión de valores o ideas objetivas, que son reproducidas después en los discursos creados por los propios sujetos en Internet.

En este sentido, la socialización puede ser entendida como el proceso mediante el cual los individuos obtienen conocimientos, habilidades y disposiciones (hábitos, creencias, actitudes y motivos) que les permiten participar como miembros de un grupo - de la sociedad-de forma más o menos efectiva (Brim, 1966) y que, a su vez, les permiten interactuar en el espacio virtual. Aunque desde la perspectiva psicológica tradicional el concepto de socialización se ha centrado en el estudio de la niñez y en el análisis del modo de aprendizaje propio de esa etapa -adquisición de actitudes, habilidades y comportamientos apropiados para la vida social-, en la actualidad dicho concepto tiene una acepción mucho más amplia.

De hecho, a pesar de que las teorías de desarrollo de la personalidad en términos de estadios (Kohlberg, 1992; Erikson, 1970, 1972; Freud, 1949; Piaget, 1948; 
Sullivan, 1953) han sido muy aceptadas - porque suministran explicaciones tanto de la conducta socialmente positiva como de la regresiva, como señalan Bandura y Walters (1974) - no se puede olvidar que estas acentúan la variabilidad intraindividual y tienden a minimizar la variabilidad interindividual debido a las diferencias. Es de esperar un alto grado de continuidad intraindividual en la conducta durante los sucesivos períodos de edad, porque los factores familiares, subculturales y biológicos que determinan en parte las experiencias de instrucción social del individuo tienden a permanecer relativamente constantes. Por el contrario, los enfoques en términos de aprendizaje social ponen de relieve las diferencias interindividuales y la continuidad intraindividual. Cabe recordar que en psicología general la variable intraindividual -sostenida por los estadistases aquella que se centra en el análisis de los cambios que forman parte de la individualidad del sujeto (rasgos, factores) a la luz de los diferentes estadios.

Desde esta perspectiva, se da bastante importancia a las características estructurales o a la herencia biogenética (a lo motriz) de los sujetos. Así, los estudios que centran sus premisas en los estadios focalizan, por ejemplo, en el análisis de los sujetos en diferentes etapas. Este tipo de variable permite observar si hay cambios en distintas temporalidades en relación a un rasgo de personalidad cualquiera, sin importar las variables ambientales. En cambio, la variabilidad interindividual -sostenida por la teoría del aprendizaje y el resto de las teorías ambientalistas- sería aquella que otorga mayor importancia a las influencias del ambiente que a las características estructurales. Entonces, se podría valorar cómo una determinada circunstancia ambiental provoca reacciones diferentes entre los individuos y les permite crear su propia individualidad.

A la luz de estos planteos, un mismo programa televisivo o una película, por ejemplo, no tendrían por qué influir de la misma forma en los diferentes espectadores, porque cada uno de ellos tiene un marco de referencia y una biografía que determinan su manera de socializar respecto a los medios. Desde este enfoque, no serían los medios los que socializan al sujeto, entendido como ente pasivo, sino que este, por su condición mediadora, socializa respecto a los medios. Así planteado, los sujetos nunca deberían ser vistos como entes pasivos, pues cuentan con un marco de referencia que les hace interactuar con universos simbólicos y trayectorias biográficas propias. Si bien en la participación virtual el sujeto crea de manera efectiva y construye relatos, en la recepción también se dan procesos de socialización, puesto que los saberes y los procesos experimentados por los receptores son reincorporados y reutilizados en la vida cotidiana.

El proceso de socialización no solo se centra en la niñez o la adolescencia, sino que se extiende a lo largo de todo el ciclo de vida e incluye todos los procesos mediante los cuales el individuo adquiere los distintos roles sociales que va desempeñando. Además, como subrayan Bandura y Walters, los individuos experimentan diversas contingencias de refuerzo y están expuestos a modelos sociales muy diferentes. Por ello se puede afirmar que, pese a la similitud biológica, existen vivencias muy diferentes entre las personas debido a sus diferentes experiencias de instrucción o aprendizaje, de manera que las tipologías de personalidad, asumiendo las premisas sobre la diferencia, carecerían de valor teórico.

En el ámbito mediático esto se hace evidente en relación a la cercanía entre los sujetos. Queda claro que en función del acceso o de la forma en que los modelos son presentados tendrán una incidencia mayor o menor. La teoría del aprendizaje social reviste gran interés, ya que se acerca al modo en que los medios influyen en los procesos de identificación y en las pautas de conducta social (Bandura y Walters 1974, p. 59). A esto responde que el proceso de adquisición de roles se vea influido por diversos agentes a lo largo de las distintas etapas del ciclo de vida del individuo, entre los que se destacan los medios de comunicación. 


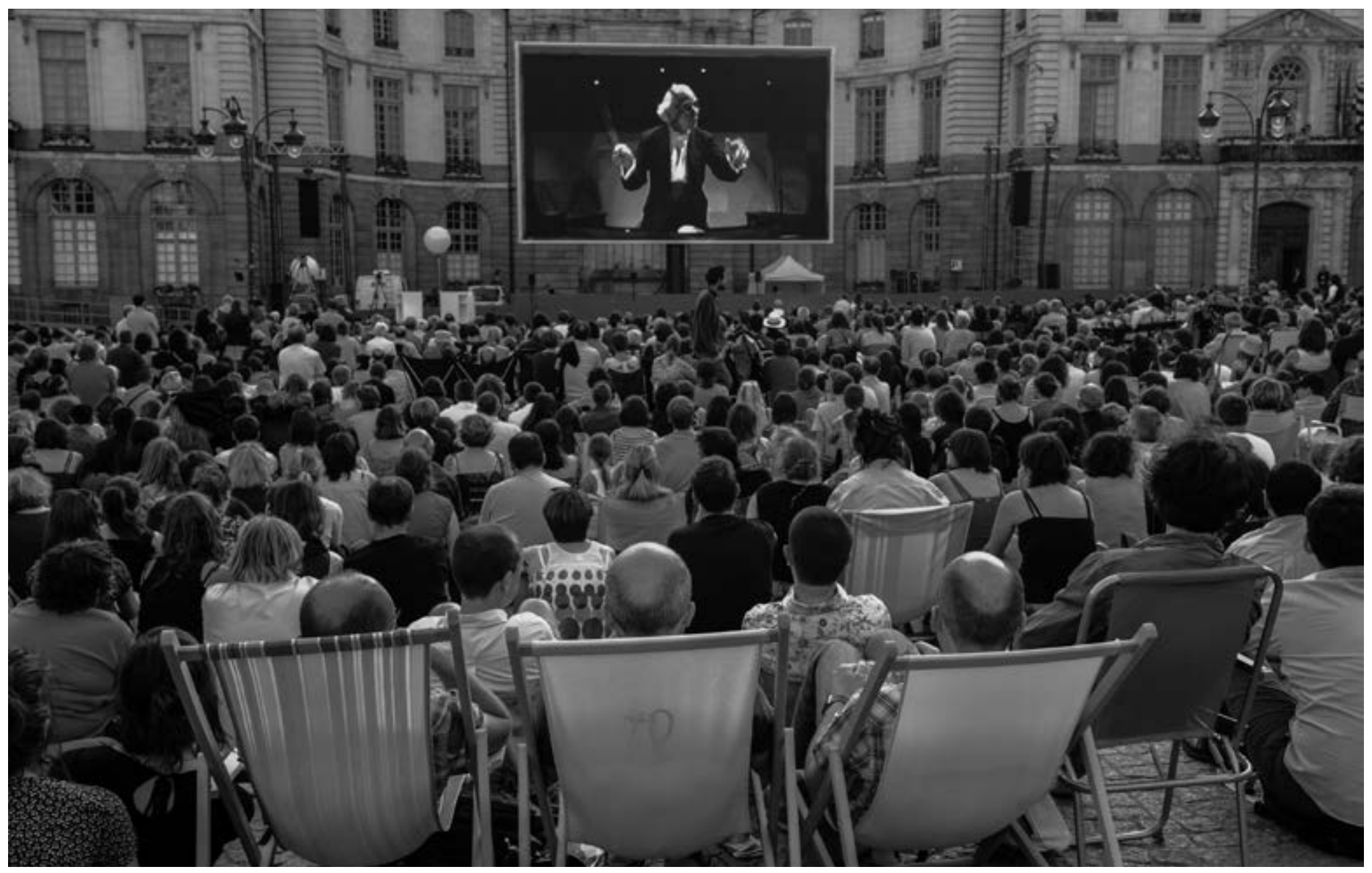

Foto: ${ }^{\circ} A F P / F r a n k$ Perry. Rennes, junio de 2011

2:: Se utiliza culturalista para abreviar Teoría de Cultura y Personalidad. El término 'culturalismo' fue empleado por primera vez en los años cincuenta y sesenta, a propósito de los trabajos norteamericanos sobre las relaciones entre cultura y personalidad, aunque los primeros de esta línea se remontan a los años treinta, con las obras de Ruth Benedict o Margaret Mead, y a los cuarenta, con las controvertidas y criticadas obras de Ray Linton y de Abraham Kardiner (Bonte e Izard, 1996).
Si bien en las primeras etapas de la vida serán los iguales (padres o amigos) los agentes que representen mayor autoridad, los medios de comunicación irán asumiendo un rol preponderante que no se puede ignorar (Moschis y Churchill, 1978). En última instancia, debe tenerse presente que los medios son, en efecto, instituciones socializadoras que influyen de forma continuada en la adopción de comportamientos, habilidades o disposiciones, en la socialización, la adquisición de roles y en los procesos de identificación (Ortiz \& Harwood, 2007; Singhal, Cody, Rogers y Sabido, 2004) que tienen hoy un papel fundamental. Esto es especialmente significativo en redes sociales como Facebook, que dan cabida a comunidades de pertenencia, o blogs temáticos y foros donde los usuarios pueden compartir sus inquietudes y dar respuesta a cualquier interrogante de la vida cotidiana. Asimismo, YouTube se ha convertido en una fuente de información y proyección de todo tipo de actividades -desde las más básicas a las más complejas-donde los internautas pueden aprender tareas, adoptar habilidades y reproducir roles.
El enfoque culturalista ${ }^{2}$ : Claudio Esteva Fabregat La perspectiva que se expone a continuación es sostenida por los autores de la llamada Escuela de Cultura y Personalidad -o antropología psicológica- que es quizás una de las primeras que se centra en el individuo como objeto de estudio directo en la práctica antropológica. Vale recordar que, a partir de los años sesenta, autores como Edwar Sapir, Bronislaw Malinowski o Géza Röheim aportaron visiones completamente nuevas, que transitan la antropología psicológica: suma de la cognitiva, lingüística o simbólica. Heredero de las aportaciones de Franz Boas -fundador de la antropología moderna norteamericana-, Claudio Esteva Fabregat es quizás el antropólogo cultural actual más representativo de esta corriente. Desde su perspectiva:

En todas las comunidades humanas existen métodos de socialización que tienen como fin orientar al individuo para su comunicación con los demás miembros de su grupo o grupos de relación, mientras al mismo tiempo le identifican con sus objetivos (Esteva Fabregat, 1993, p. 27). 
Estos métodos de socialización en forma de ideología (eidos) tienen un papel restrictivo que se impone a los miembros de cada sociedad a través de los sistemas de rol o estatus y guían la acción social. La conducta social sería entonces el resultado no solo de unas condiciones de vida, sino también, y principalmente, de la influencia de una serie de instituciones que con sus técnicas económicas rigen las actividades sociales y provocan, a su vez, deseos y frustraciones en los individuos y grupos. A juicio de Esteva Fabregat (1993):

Toda estructura de personalidad tiene como punto de referencia una organización o naturaleza que es modelada por cada sociedad según las necesidades de su estructura social, necesidades que están articuladas por medio de una cultura que define (p. 34).

Es así como debe entenderse que la cultura mediática proporciona a los individuos y a los grupos conocimientos sobre el carácter de los símbolos, la ideología y la concepción del mundo propias de una comunidad.

No cabe duda de que la cultura es una forma reguladora del comportamiento social, ya que brinda a los sujetos recursos cognitivos que contribuyen al diagnóstico de la estructura del self de los miembros de un grupo humano. La biografía de un individuo se teje entonces gracias a la historia de los demás individuos o grupos. No obstante, representa también una técnica de control de la personalidad, ya que produce ideas, bienes materiales y espirituales, y proporciona modelos de acción y valores esenciales para la vida comunitaria. En efecto, las relaciones interpersonales constituirían el método básico de comunicación y transmisión de la cultura y, por tanto, de las formas de vida que se desprenden o se aprehenden gracias a ella.
La cooperación entre individuos sería, en este sentido, fruto de los fines, aspiraciones y motivaciones comunes compartidos y transmitidos por los medios. Ahora bien, la transmisión ya no se produce de manera unidireccional, ni de arriba abajo, sino en una suerte de explosión en todas las direcciones, ya que los medios tradicionales se retroalimentan de los contenidos aparecidos en Internet y viceversa. Muchos noticiarios televisivos incluyen ahora secciones dedicadas a las redes sociales, donde exponen noticias sobre videos virales o historias que surgen en el espacio virtual.

Es importante matizar que Esteva se desmarca de las primeras conceptualizaciones culturalistas sobre la personalidad - propuestas por Ruth Benedict (1971), Margaret Mead (1972) o Abraham Kardiner (1945)que describen la personalidad como una entidad típica. Desde la perspectiva de estos antropólogos, las diferentes culturas o sociedades engendrarian diferentes tipologías de individuos o aspectos concretos de comportamiento social. Autores como Kardiner llegan a sostener que la acción de las instituciones sobre los individuos generaría una estructura de personalidad básica. A su entender, existiría un conjunto de caracteres generales, psíquicos y de comportamiento comunes a todos los miembros de una sociedad (Kardiner, 1945, p. 37), que forman el sistema proyectivo del individuo, producen la personalidad básica y son estables y permanentes. Si bien podria pensarse que las instituciones condicionan el comportamiento social de los individuos de una comunidad, no es posible estar de acuerdo con Kardiner. El impacto de las instituciones sobre la personalidad no es absoluto y varía en función de la capacidad del sujeto para establecer formas de respuesta diferentes. Precisamente a través de los nuevos medios de comunicación se abre la posibilidad de que los sujetos y los grupos puedan desarrollar identidades dobles (Turkle, 2005, 2011), alternativas o de resistencia (Sampedro, 2004a, 2004b). 
Podría decirse, en todo caso, que la personalidad es una construcción en la que se integran valores transmitidos por las instituciones que son compartidos por los miembros de una comunidad. Ahora bien, no necesariamente son reinterpretados por todos ellos de manera idéntica. En este sentido, es claro que pueden existir semejanzas entre individuos en cuanto a su manera de integrar valores transmitidos por las instituciones religiosas o mediáticas, por ejemplo, que forman parte de su contexto o marco de referencia, pero no estructuras prefijadas que puedan determinar de manera absoluta su comportamiento.

Puede así esbozarse que la cultura mediática impulsa formas de socialización condicionadas por las narrativas propuestas, que a su vez condicionan la personalidad, pero sin caer en el determinismo. No obstante, el comportamiento humano debe ser estudiado en relación a los patrones culturales transmitidos por la sociedad y por los medios, ya que, en muchas ocasiones, tanto las decisiones tomadas por los individuos como las formas de socialización se rigen más por las influencias culturales que por las decisiones personales. Sin embargo, la flexibilidad ofrecida por estas nuevas formas de comunicación y socialización mediática terminan provocando que los sujetos y los grupos puedan ser mucho más conscientes de sus identificaciones y, por tanto, tener más facilidad para moldearlas y modificarlas.

El enfoque antropológico-filosófico: Lluís Duch A diferencia de los tres autores ya examinados, Duch introduce una distinción entre las diversas instituciones socializadoras, que permite una comprensión más completa del proceso de socialización mediática. Para él, existe una serie de recursos, llamados estructuras de acogida, que son esenciales para la constitución de lo humano. Estas estructuras son las diversas instancias que, en las sucesivas etapas de la vida y en los diferentes niveles del desarrollo físico y mental del individuo, "acogen al ser humano y le permiten que sea capaz de empalabrarse él mismo y también de empalabrar -constituir- la realidad" (Duch, 2010, pp. 119-120). Una de las funciones principales de estas estructuras sería, entonces, de carácter transmisor y comunicativo, ya que permiten develar la forma de vivir propia y el desarrollo armonioso de las diversas etapas de los humanos.

Todo se inicia en la observación de que las estructuras de acogida permiten a los seres humanos pasar del caos al cosmos y de la naturaleza a la cultura, pero situando siempre al hombre en el centro. Y es que, en efecto, desde su nacimiento el ser humano necesita ser acogido, pues poco a poco socializa y va construyendo sus identificaciones en un proceso inacabado, ya que es un animal no fijado, como diría Nietzsche. Duch (2010) reclama una visión flexible de la sociedad, en la que ni esta ni sus miembros pueden ser entendidos en términos de rígidas estructuras apriorísticas, ya que forman siempre "parte de un proceso con un conjunto de etapas provisionales e históricamente indeterminadas” (p. 119). Para este autor, los sistemas genéticos y culturales son abiertos, influenciables, creativos y no determinados de antemano. En este sentido, las estructuras de acogida son elementos relacionales que, en y desde el presente de los individuos y las colectividades, les permiten a estos establecer unas vinculaciones creativas con un pasado que no se ha agotado por completo.

Según la tipología ofrecida por Duch, existen cuatro estructuras de acogida que, gracias a las acciones comunicativas, se ponen en movimiento y evidencian la inherente polifonía del ser humano. La primera es la co-descendencia, que se podría resumir con el concepto de 'familia' y que para el autor es "el lugar privilegiado donde el ser humano suele aprender a configurar, interiorizar y aplicar la 'gramática de los sentimientos'” (Duch, 2010, p. 128). La segunda es la co-residencia, que podría sintetizarse con el término 'ciudad', donde se encuentra la vivienda y donde se 


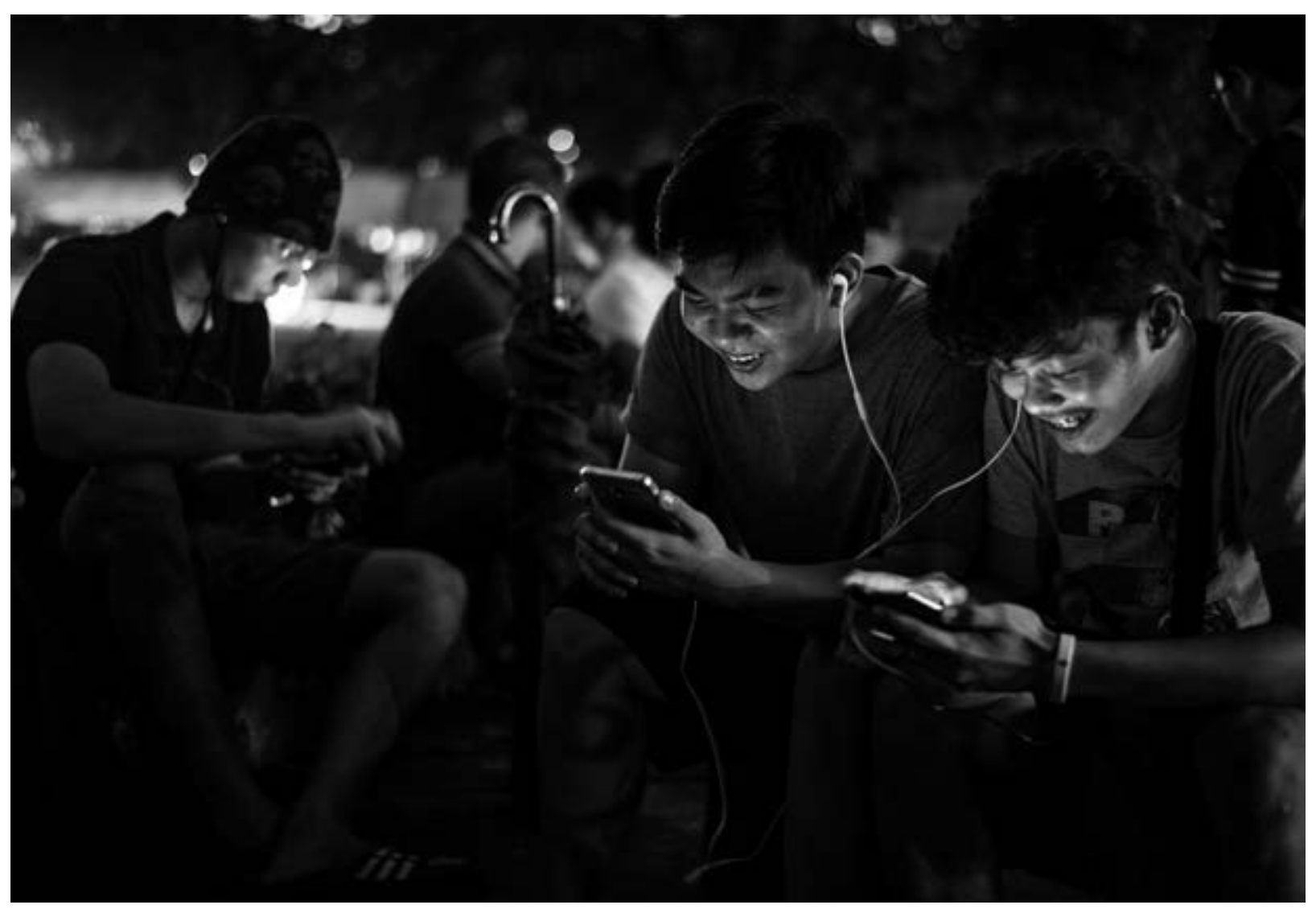

Foto: ${ }^{\circledR A F P / N o e l ~ C e l i s . ~ M a n i l a, ~ a g o s t o ~ d e ~} 2016$

hallan las instituciones significantes como la escuela, la universidad, la política, el trabajo, las asociaciones culturales y las deportivas. La co-trascendencia es la tercera de las estructuras, y se podría condensar con el concepto de 'religión', que para el autor "refiere a aquella acogida y reconocimiento que hace posible que, al margen de las convicciones personales de cada uno, individuos y grupos humanos se agrupen alrededor de un mismo tipo de creencias y de prácticas simbólicas” (Duch, 2010, pp. 154-155).

La cuarta y última, la co-mediación, es la que reviste mayor interés en el desarrollo de esta propuesta de análisis. La co-mediación establece que los medios han adquirido un rol protagonista en la socialización. Es más, estos actúan cada vez más como contexto dominante no solo de las prácticas culturales de comunicación y transmisión, sino también en sectores de la existencia humana que están vinculados con la intimidad de las personas, con sus sueños y fantasías y con su visión del mundo, como ha sugerido Duch.
A esto responde que los medios tengan una importancia crucial en la construcción de los individuos y grupos.

[Los medios] se han convertido en las entidades transmisoras más importantes de nuestra sociedad, a menudo con un carácter incluso monopolista que, subrepticiamente, tiende a desarticular y ridiculizar la labor de las tres estructuras de acogida clásicas (Duch, 2010, p. 160).

No cabe duda de que los discursos mediáticos - sean reproducidos en los medios tradicionales o creados por los usuarios en la red 2.0- se encuentran bañados de poder e inspiran una confianza y credibilidad absoluta en muchos sentidos. En palabras de Duch (2010):

La televisión articula una 'visión del mundo' que tiene -y esto suele ser frecuente en las formas de vida basadas exclusivamente en la emocionalidadun carácter imperialista y excluyente de todas las demás propuestas de vida (p. 161). 
Por tanto, cualquier clase de sentimientos, afectos, aberraciones y asuntos familiares, sin ningún tipo de pudor, son susceptibles de ser comercializados y consumidos en los platós de televisión, así como compartidos y proyectados en los muros de Facebook o en las páginas de Instagram.

Sin embargo, el verdadero problema es que las tres estructuras de acogida clásicas han sido desplazadas por la co-mediación, lo que relegó muchos de los valores positivos, como la confianza, la compasión y la amistad, que la co-descendencia, la co-residencia y la co-trascendencia transmitían. Observaciones parecidas pueden hallarse desde los estudios de comunicación. Precisamente, la hegemonía de los medios como agentes socializadores ya fue puesta de manifiesto por la Escuela de Annenberg en la década de los ochenta, a partir del pensamiento de George Gerbner, quien considera que los medios están desplazando a las tradicionales instancias socializadoras como la familia, la Iglesia o la escuela. A este respecto, tal como sostiene este autor, la preponderancia de cualquiera de las estructuras de acogida no tendrá buenos resultados (Gerbner, Gross, Signorielli y Morgan, 1980). Por eso, quizás la complementariedad sea la vía más oportuna para que el ser humano adquiera su socialización de manera efectiva y satisfactoria. En fin, que el imperio de cualquier clase de discurso nunca es bueno, del mismo modo que el predominio de una sola estructura tampoco lo es.

De esta sumaria panorámica conceptual, resulta evidente que los medios ocupan un lugar fundamental en los procesos de socialización e identificación de los individuos, pero queda claro que son los sujetos y los grupos los que se sitúan en el centro del proceso, y socializan y son socializados por los medios gracias a su condición mediadora fundamental.

\section{Conclusiones}

Desde los estudios de comunicación se ha marcado, tradicionalmente, una diferencia sustancial entre la recepción clásica y la moderna participación virtual. Se afirma que se trata de dos fenómenos por completo diferentes, y en parte lo son, debido a la interactividad. El primero, catalogado muchas veces de "antiguo", plantea que los sujetos son entes pasivos; mientras que en el segundo, considerado "nuevo", los sujetos son activos, casi hiperactivos, en la medida en que crean y recrean discursos sin fin o interactúan con otros, reales o virtuales. Ahora bien, a la luz de las teorías revisadas es posible manifestar que, si bien la interactividad trae consigo fenómenos totalmente nuevos -como la creación de avatares, por ejemplo-, la distinción entre sujetos pasivos y activos no es tan adecuada.

El ser humano conoce y construye la realidad cotidiana mediante símbolos, mitos, metáforas... en fin, mediaciones. Esto se debe a que tiene una condición mediadora fundamental que se encuentra en la base de su necesidad de socializar e interactuar, y que se da tanto en la recepción como en la participación virtual. Además, debe tenerse presente que toda socialización -sea interactiva o no- es eminentemente simbólica, en tanto representa y define en forma de poder, rol y estatus. Por eso, resulta oportuno referirse a los procesos de socialización mediática como fenómenos individuales y colectivos que se nutren de las figuraciones mediáticas, tanto de las "clásicas" como de las "nuevas".

Las teorias de los efectos en general aportan visiones limitadas, ya que reducen el proceso al impacto, a corto o largo plazo, que los contenidos o las formas de recepción o participación generan en los individuos o los grupos. De hecho, la socialización es 
clasificada en términos de estructuras o tipologías específicas de género, edad o nación. Si bien estas variables son pertinentes y ofrecen resultados acerca del impacto de los medios sobre determinados colectivos, desde este ensayo se pretende devolver a los sujetos un rol más activo, ya que ellos son, al fin y al cabo, los protagonistas de la socialización mediática, independientemente de su condición.

A este respecto, es crucial tender puentes con otras disciplinas que colocan en el centro al sujeto y lo distancian de su pasividad frente a los medios. No son los medios los que impactan sobre las personas, dado que estas necesariamente establecen un diálogo con los relatos e imágenes representadas en ellos. Justamente son estos sujetos los que crean mundos de la vida, acervos de conocimiento y universos simbólicos, que están en la base de los nuevos discursos creados en la realidad virtual.

Para estudiar la participación virtual es preciso tener presentes las experiencias que los sujetos viven de forma indirecta a través de la televisión o el cine, por ejemplo, porque ambas se realimentan de manera dialéctica. No se tratan de fenómenos aislados y, por tanto, es necesario estudiarlos en su co-implicación.

Los medios de comunicación son instituciones socializadoras o estructuras de acogida gracias a las cuales el ser humano puede aprender y comprender su entorno, pero, sobre todo, son sistemas abiertos, influenciables y creativos, que se construyen gracias a las proyecciones de los sujetos. Es decir, no son solo los medios los que proponen maneras de significar la realidad y tipificaciones acerca de lo que es el yo y lo que son los otros: ahora son también los sujetos y los grupos los que utilizan a los medios para significar la realidad.

\section{Referencias}

Bandura, A. y Walters, R. H. (1974). Aprendizaje y desarrollo de la personalidad. Madrid: Alianza Universidad.

Bandura, A. (2009). Social Cognitive Theory of Mass Communication. Bryant, J. y Oliver, M. B. (Eds.). Media Effects. Advances in Theory and Research (pp. 94-124). Nueva York: Routledge.

Bandura, A. (1962). Social Learning through Imitation. Jones, M. R. (Ed.). Nebraska Symposium on Motivation (pp. 211-269). Lincoln: University of Nebraska Press.

Bandura, A. (1977). Social Learning Theory. Englewood Cliffs: Prentice Hall Publishing.

Barber, N. A. (2014). Investigating the Potential Influence of the Internet as a New Socialization Agent in Context with Other Traditional Socialization Agents. Journal of Marketing Theory \& Practice, 21(2), 179-194.

Benedict, R. (1971). El hombre y la cultura: Investigación sobre los orígenes de la civilización contemporánea. Barcelona: Edhasa.

Berger, P. L. y Luckmann, T. (2008). La construcción social de la realidad. Buenos Aires: Amorrortu.

Bonte, P. e Izard, M. (1996). Diccionario de etnología y antropología. Madrid: Akal.

Boulier, D. (1991). Les styles de relation à la televisión. París: Réseaux, Hors Série.

Brim, O. G. (1966). Socialization through the Life Cycle. Brim, 0. G. y Wheeler, S. (Eds.). Socialization after Childhood: Two Essays (pp. 1-49). Nueva York: John Wiley \& Sons.

Bush, A. J., Smith, R. y Martin, C. (2013). The Influence of Consumer Socialization Variables on Attitude toward Advertising: A Comparison of African-Americans and Caucasians. Journal of Advertising, 28, 13-24. doi: 10.1080/00913367.1999.10673586

Chaffee, S. H., McLeod, J. M. y Walkman, D. B. (1973). Family Communications patterns an adolescents' political participation. Denis, J. (Ed.). Socialization to Politics: A reader (pp. 349-364). Nueva York: Wiley.

Chapin, J. R. (2000). Adolescent Sex and Mass Media: A developmental Approach. Adolescence, 35, 799-811. 
Collins, R. L. (2011). Content Analysis of Gender Roles in Media: Where Are We Now and Where Should We Go?. Sex Roles, 64, 290-298. doi:10.1007/s11199-010-9929-5

Dietz, T. L. (1998). An Examination of Violence and Gender Role Portrayals in Video Games: Implications for Gender Socialization and Aggressive Behavior. Sex Roles, 38, 425-442. doi:10.1023/A:1018709905920

Dill, K. E. y Thill, K. P. (2007). Video Game Characters and the Socialization of Gender Roles: Young People's Perceptions Mirror Sexist Media Depictions. Sex Roles, 57, 851-864. doi:10.1007/s11199-007-9278-1

Duch, L. (2010). Religió i comunicació. Barcelona: Fragmenta.

Durham, M. G. (1999). Girls, Media and the Negotiation of Sexuality: A study of Race, Class, and Gender in Adolescent Peer Groups. Journalism and Mass Communication Quarterly, 76 (2), 193-216.

Erikson, E. H. (1970). Infancia y sociedad. Buenos Aires: Hormé. Erikson, E. H. (1972). Sociedad y adolescencia. México: Siglo XXI.

Esteva Fabregat, C. (1993). Cultura, sociedad y personalidad. Barcelona: Anthropos.

Fisherkeller, J. E. (2002). Growing up with Television. Everyday Learnig among Young Adolescents. Filadelfia: Temple University Press.

Freud, S. (1949). An Outline of Psychoanalysis. Nueva York: Norton.

Fujioka, Y. y Austin, E. W. (2002). The relationship of Family Communication Patterns to Parental Mediation Styles. Communication Research, 29, 642-665.

Hartmann, T. y Klimmt, C. (2006). Gender and Computer Games: Exploring Females' Dislikes. Journal of Computer-Mediated Communication,11, 910-931. doi:10.1111/j.10836101.2006.00301.x

Hardin, M. y Greer, J. D. (2009). The Influence of Gender-role Socialization, Media Use and Sports Participation on Perceptions of Gender-Appropriate Sports. Journal of Sport Behavior, 32 (2), 207-226

Gerbner, G., Gross, L., Morgan, M. y Signorelli, N. (1986). Growing up with television: the cultivation perspective. Bryant, J. y Zillmann, D. (Eds.). Media effects: advances in Theory and Research (pp.17-41). Hillsdale: Lawrence Erlbaum Associates.
Gerbner, G., Gross, L., Signorielli, N. y Morgan, M. (1980) Aging with Television: Images on Televisión Drama and Conceptions of Social Reality. Journal of Communication, 30 (1), 37-47.

Gross, L. (1991). Sexual minorities and the mainstream. Journal of Homosexuality, 21, 19-46. doi: 10.1300/J082v21n01_04

Kardiner, A. (1945). El individuo y su sociedad. México: Fondo de Cultura Económica.

Kohlberg, L. (1992). Psicología del desarrollo moral. Bilbao: Desclée de Brouwer.

Lull, J. (1980). The social uses of television. Human Communication Research, 6, 197-209.

Martín Barbero, J. (1998). De los medios a las mediaciones. México: Gustavo Gili.

Martín Serrano, M. (1987). La mediación social. Madrid: Alianza.

Maturana, H. y Varela, F. (1973). De máquinas y seres vivos: Una teoría sobre la organización biológica. Santiago de Chile: Editorial Universitaria.

Mead, M. (1972). Adolescencia, sexo y cultura en Samoa. Barcelona: Laia.

McDevitt, M. y Chaffee, S. (2000). Closing Gaps in Political Communication and Knowledge: Effects of a School Intervention. Communication Research, 27, 259-292.

Moschis, G. P. y Churchill, G. A. (1978). Consumer Socialization: A Theoretical and Empirical Analysis. Journal of Marketing Research, 15 (4), 599-609.

O'Keefe, G. y Reid-Nash, K. (1987). Socializing Functions. Berger, C. y Chaffee, S. H. (Comps.). Handbook of Communication of Science (pp. 419-445). Newbury Park: Sage.

Ortiz, M. y Harwood, J. (2007). A Social Cognitive Theory Approach to the Effects of Mediated Intergroup Contact or Intergroup Attitudes. Journal of Broadcasting and Electronic Media, 51(4), 615-631.

Pasquier, D. (1996). Teen Series' Reception: Television, Adolescence and Culture of Feelings. Childhood: A Global Journal of Child Research, 3 (3), 351-373.

Piaget, J. (1948). The Moral Juggement of the Child. Nueva York: Free Press

Pindado, J. (2003). El papel de los medios de comunicación en la socialización de los adolescentes de Málaga. (Tesis Doctoral). Universidad de Málaga, España. 
Pindado, J. (2005). Los medios de comunicación en la socialización adolescente. Telos, 62, 14-20.

Potter, J. (1996). Representing reality. Discouse, rethoric and social construction. Londres: Sage.

Rodríguez, M. (1997). Enculturación y socialización. Aguirre Baztán, Á. (Ed.). Cultura e identidad cultural. Introducción a la antropología (pp. 87-106). Barcelona: Editorial Bardenas.

Sampedro, V. F. (2004a). Identidades mediáticas e identificaciones mediatizadas. Visibilidad y reconocimiento identitario en los medios de comunicación. Revista CIDOB d'Afers Internacionals, 66-67, 135-149.

Sampedro, V. F. (2004b). Identidades mediáticas. La lógica del régimen de visibilidad contemporánea. Sphera Pública, 4, 17-35.

Schütz, A. (1972a). Fenomenología del mundo social. Psicología social y sociología. Buenos Aires: Paidós.

Schütz, Alfred (1972b). La construcción significativa del mundo social. Buenos Aires: Paidós.

Schütz, A. (2003). El problema de la realidad social. Buenos Aires: Amorrortu Editores.

Schütz, A. y Luckmann, T. (2001). Las estructuras del mundo de la vida. Buenos Aires: Amorrortu Editores.

Signorelli, N. (1990). Television's mean and dangerous world: A continuation of the cultural indicators perspective. Signorielli, N. y Morgan, M. (Eds.). Cultivation analysis: New directions in a media effects research (pp. 85-106). Newbury Park: Sage.

Signorelli, N. (1993). Television and adolescents' perceptions about work. Youth \& Society, 24 (3), 314-341.

Silverstone, R., Hirsch, E. y Morley, D. (1996). Tecnologias de la información y de la comunicación y la economía moral de la familia. Silverstone, R. y Hirsch, E. (Eds.). Los efectos de la nueva comunicación (pp. 319-328). Barcelona: Bosch.

Silverstone, R. (2004). ¿Por qué estudiar los medios?. Buenos Aires: Amorrortu.

Singhal, A., Cody, M. J., Rogers, E. M. y Sabido, M. (2004). (Eds.). Entertainment Education and Social Change: History, Research and Practice. Mahwah, NJ: Lawrence Erlbaum Associates.

Sola-Morales, S. (2013a). Un narcisismo radical. La creación de identificaciones en los espacios virtuales. Alaic. Revista Latinoamericana de Ciencias de la Comunicación, 19, 86-95.
Recuperado de http://www.alaic.org/revistaalaic/index.php/ alaic/article/view/516

Sola-Morales, S. (2013b) El cuerpo y la corporeidad simbólica como forma de mediación. Mediaciones Sociales. Revista de Ciencias Sociales y de la Comunicación, 12, 42-62. Recuperado de http://pendientedemigracion.ucm.es/info/ mediars/MediacioneS12/Indice/SolaMoralesS2013/solamoraless2013.html

Sola-Morales, S. (2013c) La identificación con personajes mediáticos de ficción en la recepción mediática y en la participación virtual. Poliantea. Revista Académica y Cultural Fundación Politécnico Grancolombiano, 9 (17), 109-128. Recuperado de http://journal.poligran.edu.co/index.php/ poliantea/article/view/467/429

Sullivan, H. S. (1953). The Interpersonal Theory of Psychiatry. Nueva York: Norton.

Suess, D. et al. (1998). Media use and the relationships of children and teenagers with their peer groups: A Study of Finnish, Spanish and Swiss Cases. European Journal of Communication, 13, 521-538.

Turkle, S. (2005). The Second Self: Computers and the Human Spirit. Cambridge, MA: MIT Press.

Turkle, S. (2011). Alone Together. Why We Expect More from Technology and Less from Each Other. Nueva York: Basic Books.

Wang, X., Yu, C. y Wei, Y. (2012). Social Media Peer Communication and Impacts on Purchase Intentions: A Consumer Socialization Framework. Journal of Interactive Marketing, 26 (4), 198-208. http://dx.doi.org/10.1016/j.intmar.2011.11.004 Walkerdine, V. (1993). Daddy's gonna buy you a dream to cling to: young girls and popular television. Buckingham, D. (Ed.). Reading Audiences. Young People and the Media (pp. 74-88). Manchester: Manchester University Press.

Weber, M. (1964). Economía y Sociedad. Vol. I. México: Fondo de Cultura Económica.

Wolf, M. (1992). Los efectos sociales de los media. Barcelona: Paidós.

DOI: http://dx.doi.org/10.22235/d.v0i25.1273 